\title{
Tumor necrosis factor-related apoptosis-inducing ligand mediates the resolution of allergic airway inflammation induced by chronic allergen inhalation
}

\author{
L Faustino $^{1}$, DM Fonseca ${ }^{2}$, EB Florsheim ${ }^{1}$, RR Resende $^{3}$, AP Lepique ${ }^{1}$, E Faquim-Mauro ${ }^{4}$, E Gomes ${ }^{1}$,

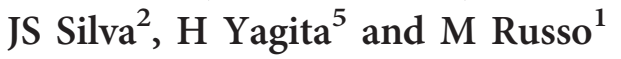

Allergic asthma can vanish over time either spontaneously or induced by allergen-specific immunotherapy. In mice with established airway allergic inflammation, chronic intranasal (IN) allergen challenges decreases progressively airway allergic inflammation. Here we compared the contribution of different regulatory pathways that could be associated with this phenomenon, known as local inhalational tolerance. We found that inhalational tolerance was not associated with increased number of regulatory Tcells or suppressive cytokines. Instead, it was associated with increased apoptosis of airway inflammatory leukocytes revealed by annexin- $V$ staining and the expression of apical caspase 8 and effector caspase 3. Also, the transition from acute to chronic phase was associated with a shift in the expression of pro-allergic to pro-apoptotic molecules. The tumor necrosis factor-related apoptosis-inducing ligand (TRAIL) was found to be a key molecule in mediating resolution of allergic inflammation because anti-TRAIL treatment blocked apoptosis and increased the infiltration of T helper type 2 (Th2) cells and eosinophils. Notably, repeated IN treatment with recombinant TRAIL in established airway allergic inflammation augmented leukocyte apoptosis and decreased the frequency of interleukin-5-producing Th2 cells and eosinophils to airways. Our data indicate that TRAIL signaling is sufficient for downmodulation of allergic airway disease, suggesting a potential therapeutic use of TRAIL for asthma treatment.

\section{INTRODUCTION}

Allergic asthma is a chronic inflammatory disease characterized by airway eosinophilia, airway hyperreactivity, mucus hypersecretion, and high titers of immunoglobulin E (IgE) (1). T helper type 2 (Th2) lymphocytes are thought to be responsible for the initiation, progression, and persistence of the disease because upon allergen challenge they secrete type-2 cytokines such as interleukin-4 (IL-4), IL-5, IL-9, and IL-13 that mediate the Th2-associated inflammatory network and IgE production. ${ }^{1-4}$ However, not all individuals develop allergy upon exposure to allergens and some patients can resolve it over time either spontaneously or by repeated allergen challenges that is the basis of specific immunotherapy. ${ }^{5-8}$ Therefore, the immune system has evolved regulatory mechanisms to control allergic responses to harmless noninfectious antigens.

Murine models have been developed to study either effector or immune regulatory mechanisms operating in allergic lung disease. ${ }^{9-14}$ One well-characterized murine model of immune regulation that leads to resolution of allergic airway inflammation consists of given long-term IN allergen challenges to sensitized mice. ${ }^{15-18}$ This phenomenon, known as local inhalational tolerance, is characterized by local resolution of allergic airway inflammation because it courses along with persistent systemic IgE production. ${ }^{19-21}$ Various studies using this model suggested different mechanisms for the resolution of allergic inflammation such as the emergence of Foxp $3{ }^{+} \mathrm{CD} 25^{+} \mathrm{CD} 4{ }^{+}$

\footnotetext{
${ }^{1}$ Department of Immunology, Institute of Biomedical Sciences, University of São Paulo, São Paulo, Brazil. ${ }^{2}$ Department of Biochemistry and Immunology, School of Medicine of Ribeirão Preto, University of São Paulo, Ribeirão Preto, Brazil. ${ }^{3}$ Department of Biochemistry and Immunology, Federal University of Minas Gerais, Belo Horizonte, Brazil. ${ }^{4}$ Laboratory of Immunopathology, Butantan Institute, São Paulo, Brazil and ${ }^{5}$ Department of Immunology, Juntendo University School of Medicine, Tokyo, Japan. Correspondence: M Russo (momrusso@icb.usp.br)
} 
regulatory $\mathrm{T}$ (Treg) cells, ${ }^{22-24}$ or regulatory B cells, ${ }^{25}$ or a population of transforming growth factor (TGF)- $\beta_{1}$-expressing macrophages. ${ }^{26}$ Although these studies were well conducted, none of them compared the specific contribution of these different regulatory pathways.

Generally, resolution of inflammation might involve suppressive mechanisms that inhibit the action of inflammatory cells and/or the release of inflammatory mediators or induction of apoptosis of effector cells. Here we evaluated whether Treg cells, suppressive cytokines, or apoptosis could be associated with the inhalational tolerance. For this, we treated mice with specific monoclonal antibodies (mAbs) to block functionally key molecules involved in regulatory/suppressor activities or apoptotic process. $\mathrm{mAb}$ directed to inactivation of Treg cell function or TGF- $\beta$ were without effect. However, neutralizing $\mathrm{mAb}$ directed to members of the tumor necrosis factor (TNF) superfamily of death-inducing ligands, such as Fas ligand (FasL) and TNF-related apoptosis-inducing ligand (TRAIL), or to programmed cell death protein-1 (PD-1), a protein of immunoglobulin superfamily, revealed that the TRAIL pathway was critically involved in the resolution of allergic inflammation induced by long-term ovoalbumin (OVA) administration given at weekly intervals.

\section{RESULTS}

\section{Resolution of allergic inflammation induced by long-term allergen challenges is restricted to the lung}

Acute exposure of sensitized mice to inhaled antigen elicits airway eosinophilia and elevation of serum IgE. Conversely, chronic allergen exposure leads to resolution of airway inflammation but persistent antibody production (Supplementary Figure S1 online), a phenomenon termed as local inhalational tolerance. ${ }^{19}$ Here we extended this observations and studied the effects of prolonged antigen exposure on two different sites of sensitized mice, the airways and peritoneal cavity. For this, we established the acute allergic and chronic tolerant protocols monitoring the eosinophilic inflammation of the airways and the systemic IgE production $24 \mathrm{~h}$ after each antigen challenges given at weekly intervals for a period of 5 weeks. As shown in Figure 1, the number of eosinophils in the bronchoalveolar lavage (BAL) fluid started to increase $24 \mathrm{~h}$ after the first OVA challenge, reaching maximum values after the third challenge, but decreasing thereafter (Figure 1a). In contrast, the serum levels of IgE increased progressively up to the second OVA challenge and remained elevated thereafter (Figure 1b). Next we compared in sensitized mice the effects of prolonged OVA challenges given concomitantly in the airways and peritoneal cavity as depicted in Figure 1c. We termed the group of mice exposed to two OVA challenges as acute and the group of mice exposed to five OVA challenges as chronic (Figure 1c). We found that the number of eosinophils increased in BAL and in peritoneal cavity after acute exposure to OVA (Figure 1d). As expected, chronic IN OVA exposure resulted in a drastic reduction of eosinophils in the BAL (Figure 1d). However, in sharp contrast with OVA airway challenges, chronic IP OVA did not decrease eosinophil influx to peritoneal cavity when
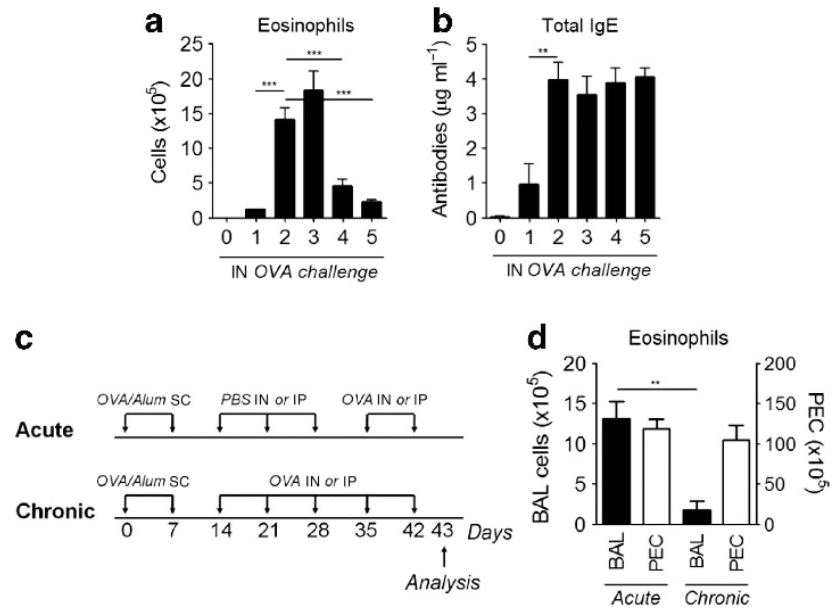

Figure 1 Resolution of allergic inflammation induced by chronic allergen challenges is restricted to the lung. (a) Time course of eosinophils influx in the bronchoalveolar lavage (BAL) and (b) production of total immunoglobulin $E(\mathrm{IgE})$ in the serum of sensitized mice $24 \mathrm{~h}$ after each intranasal (IN) ovoalbumin (OVA) challenge. (c) Protocol of OVA/Alumsensitized mice exposure acutely (acute) or chronically (chronic) to OVA. (d) Number of eosinophils in the BAL and peritoneal cavity after two (acute) or five (chronic) OVA challenges. BAL cells and peritoneal exudate cells (PEC) were harvested $24 \mathrm{~h}$ after of the last OVA challenge, on day 43. Values represent the mean \pm s.e.m. for groups of three mice and are representative of two independent experiments. Significant differences ${ }^{\star *} P<0.01,{ }^{* \star *} P<0.001$ are shown.

compared with acute OVA exposure (Figure 1d). These results clearly indicated that the resolution of allergic inflammation induced by long-term allergen challenges depends on the site where OVA is given and effectively occur at mucosal surface but not in the serosal cavity.

Treg cells and suppressive cytokines do not mediate the resolution of allergic airway inflammation

It has been reported that the resolution of allergic airway inflammation or airway hyperreactivity induced by long-term allergen challenge is associated with local accumulation of Treg cells. ${ }^{22,23}$ Therefore, we evaluated the percentage and number of Foxp $3^{+} \mathrm{CD} 4{ }^{+} \mathrm{T}$ cells in the airways and draining lymph nodes of mice that received inhaled OVA acutely or chronically. We found that Foxp $3^{+} \mathrm{CD} 4{ }^{+}$T cells were increased in the BAL and in lungs of acute but not chronic mice, whereas the number of Treg cells in the mediastinal draining lymph node was increased similarly in acute and chronic groups (Figure 2a and Supplementary Figure S2a-c) when compared with naive mice. The percentage of Foxp ${ }^{+}$Treg cells during the course of allergen challenges in the BAL increased until third challenge and decreased thereafter (Supplementary Figure S2d), indicating that airway Treg cells are associated with allergic inflammation rather than with inhalational tolerance. We also assessed the function of Treg cells in vivo by treating the animals with anti-CD25 (PC61) mAb to deplete and/or to inactivate putative $\mathrm{CD} 25^{+}$Treg cells as shown previously by us and others. ${ }^{27-30}$ During acute OVA challenge, the anti-CD25 $\mathrm{mAb}$ treatment increased the number of eosinophils in the 
a

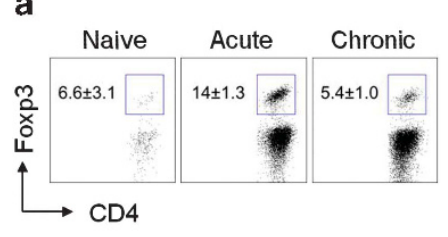

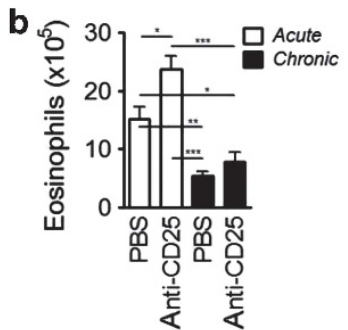
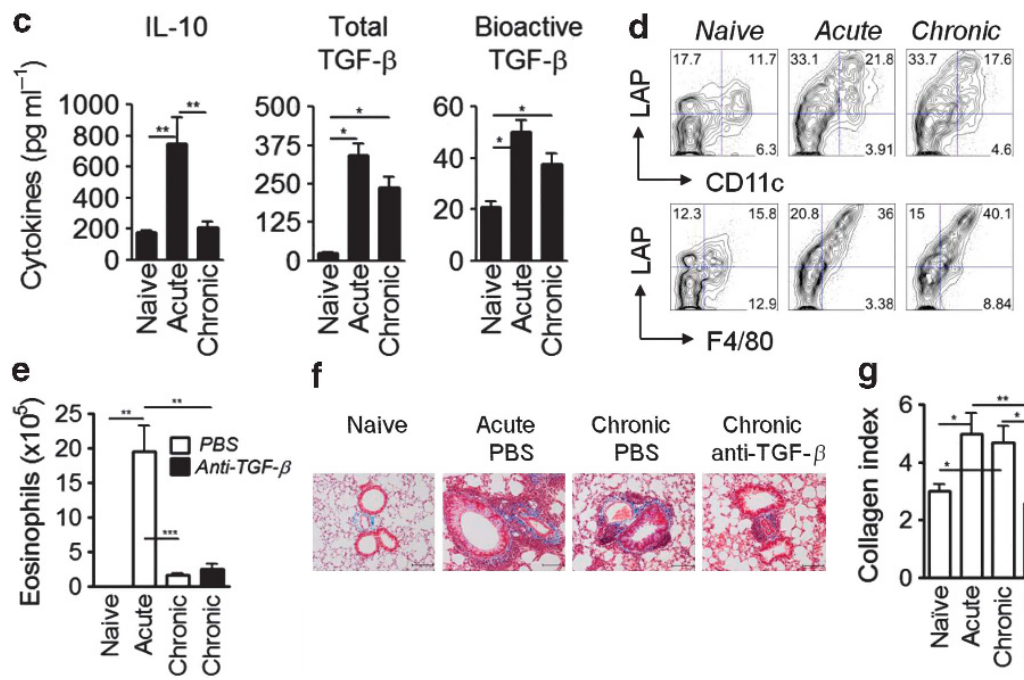

$\longrightarrow \mathrm{CD} 11 \mathrm{c}$

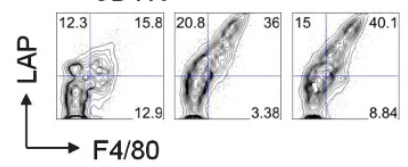

$\mathbf{f}$
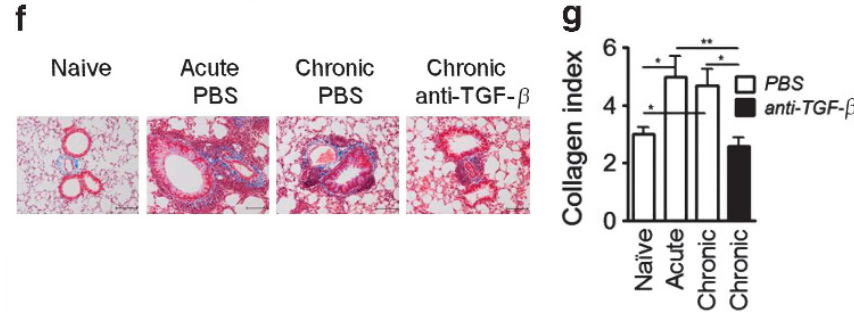

Figure 2 Regulatory T cells and suppressive cytokines do not mediate the resolution of allergic airway inflammation. (a) Percentage of bronchoalveolar lavage (BAL) Foxp3 ${ }^{+} \mathrm{CD} 4^{+} \mathrm{T}$ cells of naive, acute, and chronic mice $24 \mathrm{~h}$ after the last ovoalbumin (OVA) challenge. Cells recovered from BAL were stained for $\mathrm{CD} 3, \mathrm{CD} 4$, and Foxp3 and gated in $\mathrm{CD} 4{ }^{+} \mathrm{CD} 3{ }^{+}$cells. (b) Number of BAL eosinophils of acute and chronic mice treated with anti-CD25 monoclonal antibody (mAb) or phosphate buffered saline (PBS) IV at days 34, 35, 36, and 41. Cells were harvested $24 \mathrm{~h}$ after of the last OVA challenge, on day 43. (c) BAL levels of interleukin-10 (IL-10) and transforming growth factor (TGF)- $\beta$ determined by enzyme-linked immunosorbent assay.

(d) Expression of LAP by lung dendritic cells (CD11 $+\mathrm{MHCll}+$ ) or macrophages ( $\mathrm{F} 480+\mathrm{MHCll}+$ ) of naive, acute and chronic mice $24 \mathrm{~h}$ after the last OVA challenge. Cells recovered from lung were stained for MHCII, CD11c, F4/80 and LAP and gated in MHCII + cells. (e) Number of eosinophils in the BAL of mice treated with intranasal (IN) anti-TGF- $\beta$ mAb or with PBS. (f) Representative lung sections stained with Masson's Trichrome for analysis of collagen deposition (original magnification $\times 20$ ). $(\mathbf{g})$ Collagen index of lung sections stained with Masson's Trichrome. Values represent the mean \pm s.e.m. for groups of three mice and are representative of two independent experiments. Significant differences ${ }^{*} P<0.05$, ${ }^{* \star} P<0.01$, and ${ }^{* \star \star} P<0.001$ are shown.

BAL when compared with animals non-treated (phosphate buffered saline (PBS) group) (Figure 2b) or treated with isotype control rat IgG1 mAb (GL113) (data not shown). However, during chronic OVA challenge, the anti-CD25 treatment did not change the lung tolerant state as evidenced by reduced number of eosinophils in the BAL (Figure 2b). Altogether, these results indicate that Treg cells are not involved in the suppression of lung allergic inflammation induced by chronic OVA challenges.

Because suppression of allergic airway inflammation has also been attributed to the production of IL-10 and TGF- $\beta,{ }^{26,31,32}$ we measured the content of these suppressive cytokines in the airways. We found that the levels of IL-10 in the BAL and the frequency of IL-10-producing Foxp $3^{-} \mathrm{CD} 4^{+} \mathrm{T}$ cells, but not Foxp $3^{+}$Treg cells, in the lungs were increased during acute but not chronic OVA challenges (Figure $2 \mathrm{c}$ and Supplementary Figure S2e), indicating that IL-10 is more associated with acute allergic inflammation than local inhalational tolerance. In addition, experiments performed in IL-10-deficient mice confirmed that IL-10 is dispensable for the induction of local inhalational tolerance as IL-10-deficient mice developed it normally (Supplementary Figure S2f). Next, we measured the levels of total and bioactive TGF- $\beta$ and found that they were increased in the BAL of mice that received acute or chronic OVA challenges (Figure 2c). We also determined by flow cytometry cells expressing latency-associated peptide (LAP), which indirectly detect the TGF- $\beta$-producing cells. ${ }^{33} \mathrm{We}$ found that lung $\mathrm{CD}_{11 \mathrm{c}^{+}} \mathrm{MHCII}^{+}$and $\mathrm{F} 4 / 80^{+} \mathrm{MHCII}^{+}$, but not lung Foxp $3^{+}$or Foxp $3^{-} \mathrm{CD} 4^{+} \mathrm{T}$ cells, expressed high levels of LAP during acute and chronic OVA challenges (Figure $2 \mathbf{d}$ and Supplementary Figure S2e). Because the results with TGF- $\beta$ and LAP were not conclusive, we investigated the in vivo role of TGF- $\beta$ by treating chronic mice with two IN injections of neutralizing anti-TGF- $\beta$ mAb. We found that anti-TGF- $\beta$ treatment did not affect the tolerant state as assessed by eosinophil numbers (Figure 2e). The number of eosinophils was sixfold lower in chronic mice than in acute mice irrespective of anti-TGF- $\beta$ treatment (Figure 2e). Because TGF- $\beta$ has an important role in airway remodeling, ${ }^{34}$ we also investigated the lung collagen deposition in acute and chronic mice. Masson staining of lung sections revealed an increase of peribronchial and perivascular collagen deposition in mice with 
both acute and chronic-inhaled OVA (Figure 2f,g). Notably, lung collagen deposition was significantly inhibited in chronic mice treated with anti-TGF- $\beta$ when compared with nontreated PBS group (Figure 2f,g), indicating that the $\mathrm{mAb}$ is biologically active. We concluded that TGF- $\beta$ is not involved in inhibition of allergic inflammation. All in all, these results clearly show that Treg cells or suppressive cytokines IL-10 and TGF- $\beta$ do not mediate the resolution of allergic airway inflammation induced by long-term allergen challenge.

\section{Apoptosis of airway leukocytes increases during chronic OVA exposure}

Accumulating evidence indicate that apoptosis (programmed cell death) has a key role in the resolution of inflammatory processes and re-establishment of tissue homeostasis. ${ }^{35,36}$ Therefore, we determined annexin- $\mathrm{V}$ staining, a marker of apoptotic cells, on infiltrating airway leukocytes during acute or chronic exposure to OVA. As shown in Figure 3, the frequency of annexin $-\mathrm{V}^{+} \mathrm{PI}^{-}$apoptotic cells was higher during the chronic OVA exposure than during acute exposure. Indeed, the percentage of apoptotic cells during chronic OVA exposure was roughly twofold higher than during acute exposure (Figure 3a,b). Notably, different cell types such as $\mathrm{CD} 4^{+}$ $\mathrm{T}$ cells, eosinophils ( $\mathrm{MHCII}^{-}$Siglec- $\left.-\mathrm{F}^{+} \mathrm{Gr}^{\text {low }}\right)$, neutrophils $\left(\mathrm{MHCII}^{-}\right.$Siglec- $\left.-\mathrm{F}^{-} \mathrm{Gr} 1^{\text {high }}\right)$, and $\mathrm{MHCII}^{+} \mathrm{CD} 1 \mathrm{~b}^{+}$cells stained positively for annexin-V (Supplementary Figure S3), indicating that different cells rather than a specific cell type are undergoing apoptosis during chronic OVA. Apoptosis can be initiated by the activation of cell-surface death receptors belonging to the TNF receptor superfamily such as Fas and TRAIL receptors or by PD-1 of the immunoglobulin superfamily. ${ }^{37-39}$ Thus, we determined the expression of members of death-inducing ligands as well as pro-allergic or modulatory and effector molecules of apoptosis. As shown in Figure 3c, the mRNA expression of TRAIL (Tnfsf10) and PD-1 ligand (CD274) was higher in acute than in chronic group, whereas the expression of FasL (Tnfsf6) was higher in chronic group. Interestingly, two molecules associated with TRAIL signaling were differentially expressed in acute and chronic group. The expression of pro-allergic E3 ubiquitin ligase midline 1 (MID1) ${ }^{40}$ was highly expressed in acute group, whereas the expression of insulin-like growth factor-binding protein-3, a pro-apoptotic molecule ${ }^{41,42}$ predominated in chronic mice (Figure 3d). Finally, the expression of apical (casp8 (caspase 8)) and effector (casp3) caspases was only observed in chronic mice, whereas the expression of the initiator casp9 that is involved in the intrinsic or mitochondrially gated pathway of apoptosis ${ }^{43}$ was significantly increased in acute group (Figure 3e). Overall, the above results indicate that an active extrinsic apoptotic process is operating in chronic mice.

\section{Inhibition of apoptosis restores allergic airway inflammation}

To further investigate the apoptotic process, we treated chronic mice with five IN injections of anti-FasL, anti-TRAIL, or antiPD-1 during the last two OVA challenges. We found that antiTRAIL treatment, but not anti-FasL or anti-PD-1, markedly reduced the percentage of annexin- $\mathrm{V}^{+} \mathrm{PI}^{-}$apoptotic cells in the BAL of chronic mice when compared with PBS group (Figure 4a,b). Notably, the number of eosinophils and neutrophils increased in the BAL of chronic mice treated with anti-TRAIL when compared with those treated with antiFasL, anti-PD-1, or PBS (Figure 4c-e). The number of eosinophils found in BAL of chronic mice treated with
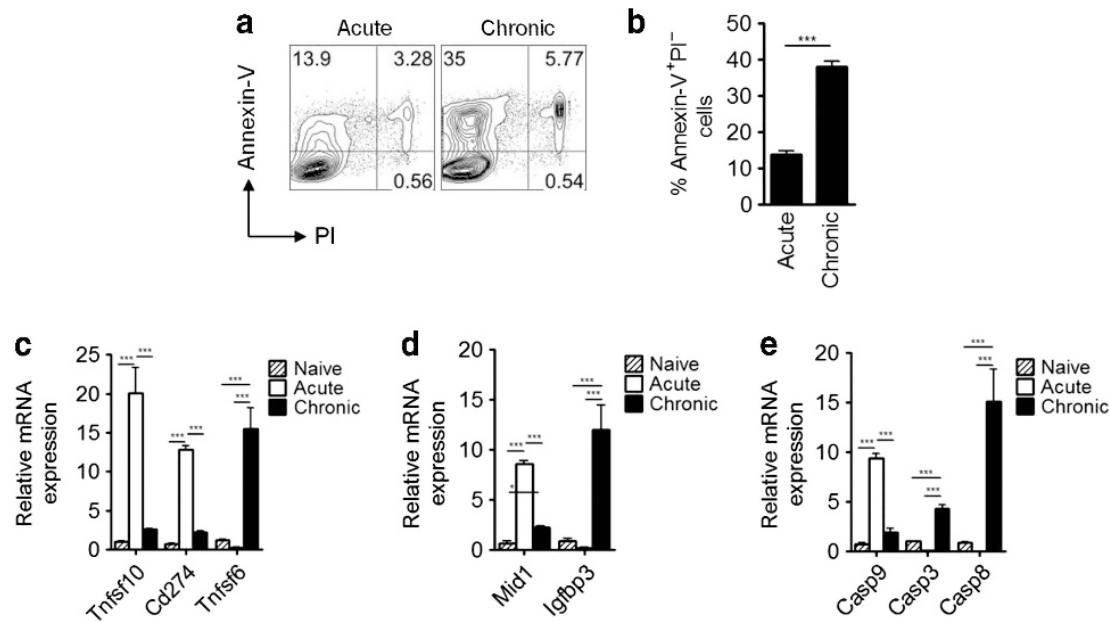

Figure 3 Apoptosis of airway leukocytes increases during chronic ovoalbumin (OVA) exposure. (a,b) Percentage of cells expressing annexin-V (apoptotic cells) and propidium iodide (PI; necrotic cells) in the bronchoalveolar lavage (BAL) of acute and chronic mice. Relative mRNA expression of (c) Tnfsf10 (tumor necrosis factor-related apoptosis-inducing ligand, TRAIL), CD274 (PD-L1), Tnfsf6 (Fas ligand, FasL), (d) Mid1 (MID1), and Igfbp3 (insulin-like growth factor-binding protein-3, IGFBP-3), and (e) casp9 (caspase 9), casp3 (caspase 3), and casp8 (caspase 8) from lungs of naive, acute, or chronic mice. Relative amounts of mRNA were normalized to $\beta$-actin RNA levels within each sample and expressed as relative target abundance by the $\Delta \Delta C T$ method. Values represent the mean \pm s.e.m. for groups of four mice and are representative of two independent experiments. Significant differences ${ }^{\star} P<0.05$, and ${ }^{\star * \star} P<0.001$ are shown. 

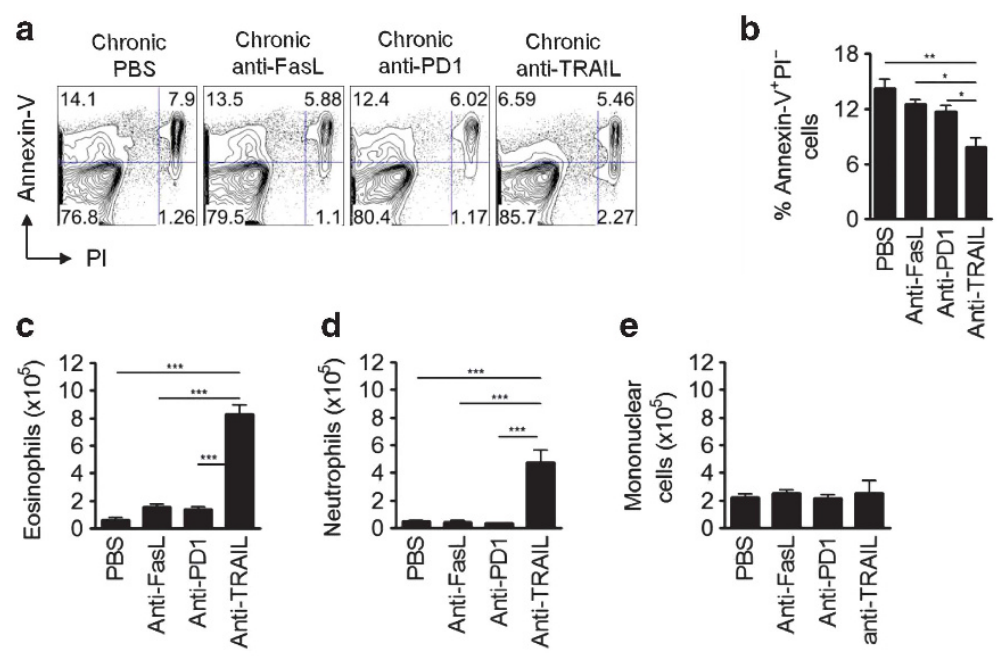

e
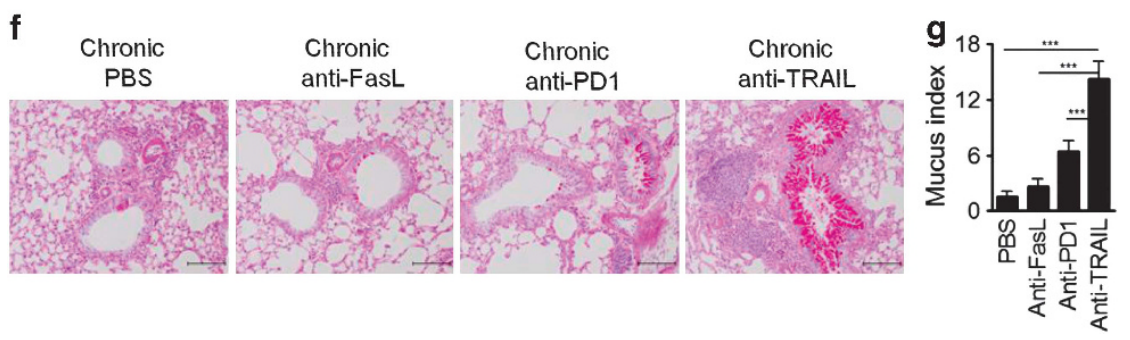

Figure 4 Inhibition of apoptosis restores airway allergic inflammation. (a,b) Percentage of cells expressing annexin- $V$ (apoptotic cells) and propidium iodide (PI; necrotic cells) in the bronchoalveolar lavage (BAL) of chronic mice treated with intranasal (IN) anti-FasL (Fas ligand), anti-PD-1, anti-TRAIL (tumor necrosis factor-related apoptosis-inducing ligand) monoclonal antibody (mAb), or with phosphate buffered saline (PBS). Number of (c) eosinophils, (d) neutrophils, and (e) mononuclear cells in the BAL. (f) Representative lung sections stained with hematoxylin/periodic acid-Schiff (PAS) for analysis of cellular inflammation and mucus production (original magnification $\times 10)$. (g) Mucus index of lung sections stained with hematoxylin/ PAS. Values represent the mean \pm s.e.m. for groups of four mice and are representative of three independent experiments. Significant differences ${ }^{\star} P<0.05,{ }^{* *} P<0.01$, and ${ }^{* *} P<0.001$ are shown.

anti-TRAIL reached similar levels to that seen during acute OVA challenge (Supplementary Figure S4a). In addition to increased number of eosinophils in BAL, lung inflammatory cell infiltrates and mucus production were higher in chronic mice treated with anti-TRAIL than those treated with antiFasL, anti-PD-1, or PBS (Figure 4f, g). We confirmed that FasL was dispensable for the resolution of allergic inflammation in OVA-sensitized mice carrying spontaneous mutations in the fas gene (Supplementary Figure S4b). These results indicate that chronic OVA challenge triggers the TRAIL pathway of apoptosis that is associated with the resolution of allergic lung inflammation.

\section{Anti-TRAIL treatment augments activated Th2 cells in the lungs of chronic mice}

Because Th2 cells orchestrate allergic airway inflammation, we next examined the impact of anti-TRAIL treatment on activated Th2 cells in the lungs of chronic mice. We found that the frequency of Foxp $3^{-} \mathrm{CD} 4^{+} \mathrm{T}$ cells expressing the activation markers CD69 and CD25 were increased in the lungs of chronic mice treated with anti-TRAIL when compared with non-treated mice (PBS group) (Figure 5a-c and Supplementary Figure S5e). The percentage of the transcription factor GATA-3 expression in lung Foxp $3^{-} \mathrm{CD} 4^{+} \mathrm{T}$ cells was not different between groups (Figure 5d). Notably, the percentage of $\mathrm{CD} 4^{+} \mathrm{T}$ cells producing IL-5, but not IL-4, was augmented in the lungs of chronic mice treated with anti-TRAIL when compared with PBS group (Figure 5e-g). Indeed, the frequency of activated Th2 cells found in anti-TRAIL-treated group was similar to that obtained in the lungs of acute mice (Supplementary Figure S5a-d). Taken together, our results indicate that blockade of TRAIL signaling during long-term allergen challenges augments activated Th2 cells in the lung and promotes allergic airway inflammation.

\section{Treatment with recombinant TRAIL augments apoptosis} and attenuates Th2-mediated allergic lung inflammation

To determine directly the anti-allergic effect of TRAIL signaling in vivo, we treated allergic mice during acute OVA challenge with four IN injections of human recombinant TRAIL (rTRAIL) that preferentially activates death receptor DR5 $5^{44}$ as depicted in Figure 6a. We found that treatment with rTRAIL increased the percentage of annexin- $\mathrm{V}^{+} \mathrm{PI}^{-}$apoptotic cells in the airways as revealed by flow cytometry analysis of BAL cells when compared with non-treated mice (PBS group) (Figure 6b,c). More importantly, rTRAIL treatment of allergic mice reduced the percentage of lung $\mathrm{CD} 4{ }^{+} \mathrm{T}$ cells producing IL-5 (Figure 6d,e) and decreased the number of eosinophils 
a

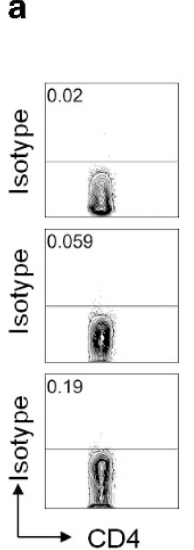

e

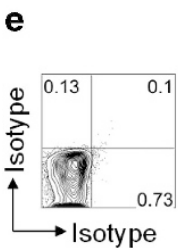

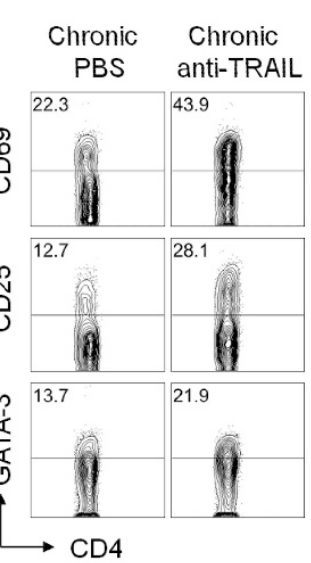
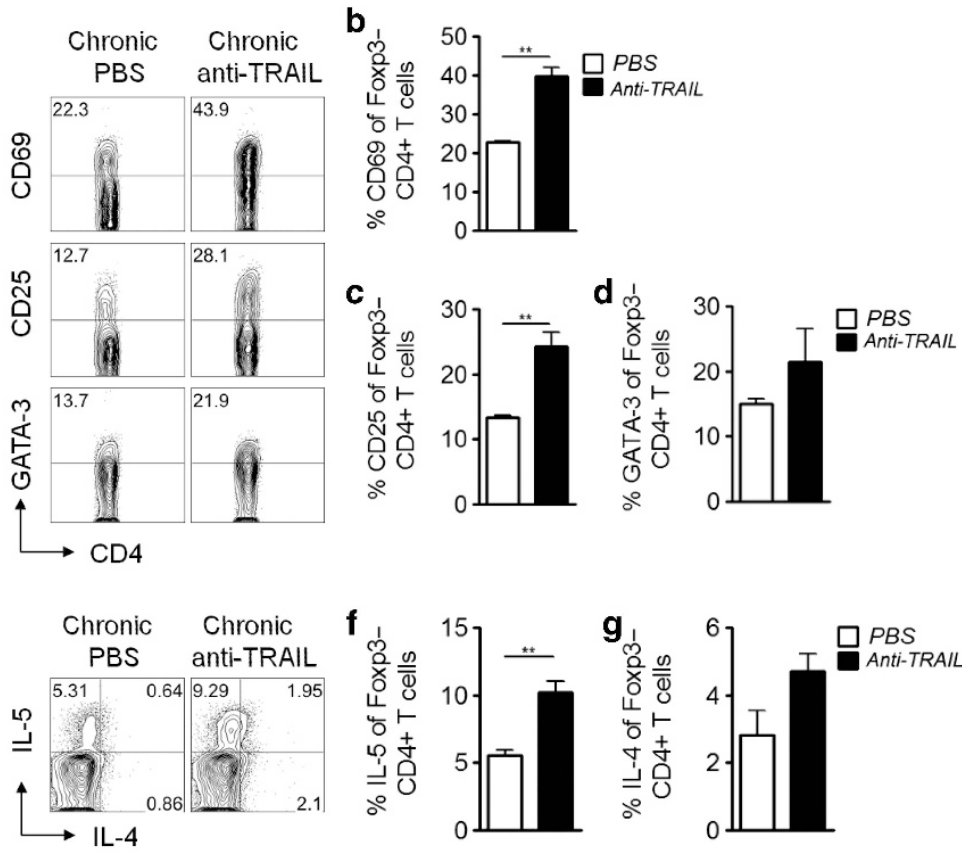

Figure 5 Anti-tumor necrosis factor-related apoptosis-inducing ligand (TRAIL) treatment augments activated T helper type 2 (Th2) cells in the lungs of chronic mice. (a-d) Percentage of Foxp3 ${ }^{-} \mathrm{CD}^{+}{ }^{+} \mathrm{T}$ cells expressing CD69, CD25, and GATA-3 in the lungs of chronic mice treated with intranasal (IN) anti-TRAIL monoclonal antibody (mAb) or with phosphate buffered saline (PBS). (e-g) Percentage of Foxp3 ${ }^{-}$CD4 ${ }^{+} \mathrm{T}$ cells expressing interleukin (IL)-5 and IL-4. Cells recovered from lungs were stained for CD3, CD4, Foxp3, CD69, CD25, and GATA-3 and gated in Foxp3 ${ }^{-} \mathrm{CD} 4^{+} \mathrm{CD}^{+}$cells. For analysis of cytokine production, lung cells were first stimulated ex vivo with PMA and ionomycin for $5 \mathrm{~h}$ in the presence of monensin in the last $3 \mathrm{~h}$ and gated on $\mathrm{CD}_{4}{ }^{+} \mathrm{CD} 3^{+}$cells for analysis of cytokine expression. Values represent the mean \pm s.e.m. for groups of four or more and are representative of three independent experiments. Significant difference ${ }^{* \star} P<0.01$ is shown.
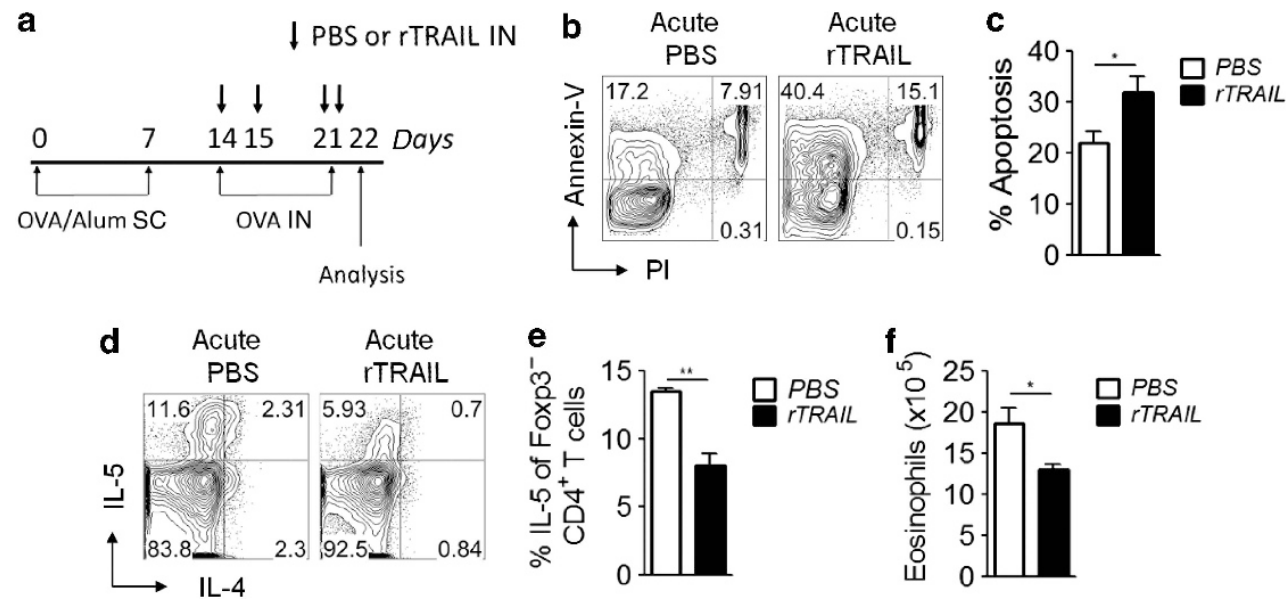

Figure 6 Treatment with recombinant tumor necrosis factor-related apoptosis-inducing ligand (rTRAIL) augments apoptosis and attenuates Thelper type 2 (Th2)-mediated allergic lung inflammation. (a) Protocol of rTRAIL treatment in ovoalbumin (OVA)-induced allergic mice. (b) Percentage of cells expressing annexin- $\mathrm{V}$ and propidium iodide (PI) in the bronchoalveolar lavage (BAL) of allergic mice treated with intranasal (IN) rTRAIL or with phosphate buffered saline (PBS). (c) Percentage of apoptotic (annexin- $\mathrm{V}^{+} \mathrm{PI}^{-}$) cells in the BAL of allergic mice treated with rTRAIL or PBS. (d,e) Percentage of Foxp3 ${ }^{-}$CD4 ${ }^{+}$T cells expressing interleukin (IL)-5 and IL-4. Cells recovered from lung were stimulated ex vivo with PMA and ionomycin for $5 \mathrm{~h}$ in the presence of monensin in the last $3 \mathrm{~h}$ and gated on $\mathrm{CD} 4^{+} \mathrm{CD} 3^{+}$cells for analysis of cytokine expression. (f) Number of eosinophils in the BAL of allergic mice treated with rTRAIL or PBS. Values represent the mean \pm s.e.m. for groups of four and are representative of three independent experiments. Significant differences ${ }^{*} P<0.05$ and ${ }^{* *} P<0.01$ are shown.

in the BAL (Figure 6f). These results reinforce the notion that TRAIL signaling acts on inflammatory cells including Th2 cells and eosinophils, thereby attenuating allergic airway inflammation.

\section{DISCUSSION}

In this study, we have investigated different regulatory/ suppressor pathways that might underline the resolution of allergic airway inflammation induced by chronic long-term 
allergen challenges. Our study confirmed and extended this notion by showing that the resolution of allergic inflammation was observed only when chronic OVA challenges were delivered at mucosal surface (airway) but not when administered into serosal peritoneal cavity, indicating that the site where OVA is given is fundamental for the resolution of allergic inflammation induced by long-term allergen challenges. The major pathways of anti-inflammatory mechanisms involve are as follows: (1) production of anti-inflammatory substances such as suppressive cytokines (IL-10, TGF- $\beta ;^{45,46}$ ), bioactive lipids (lipoxin ${ }^{47}$ and prostaglandins ${ }^{48}$ ), and endogenous mediators (glucocorticoids ${ }^{49}$ and acetylcholine ${ }^{50}$ ); (2) Induction of regulatory cells (e.g., suppressor lymphocytes such as Treg, Tr1, Th3, regulatory B, and CD8 ${ }^{+} \mathrm{T}$ cells, suppressor macrophages, and mesenchymal cells ${ }^{51-54}$; and (3) apoptosis of infiltrating inflammatory cells including effector T cells. ${ }^{35,55,56}$ All these pathways are highly regulated and implicated in resolution of inflammatory processes and re-establishment of tissue homeostasis and failures in those immunoregulatory mechanisms can result in progression and/or persistence of a range of inflammatory diseases including asthma. ${ }^{57-59}$

Because local inhalational tolerance was first attributed to the emergence and accumulation of Foxp $3^{+}$Treg cells in the airways or local draining lymph nodes, ${ }^{22-24}$ we monitored the appearance of Treg cells during acute and chronic OVA challenges. Our results showed that Foxp $3^{+}$Treg cells were by far more abundant in the airways of acute than in chronic mice, suggesting a Treg-independent mechanism for inhalational tolerance. Also, depletion and/or inactivation of putative $\mathrm{CD} 25^{+}$Treg cells by an effective anti-CD25 treatment ${ }^{27-30}$ did not restore airway eosinophilia of chronic OVA group. In contrast, anti-CD25 treatment exacerbated allergic airway inflammation of acute OVA group. These results are in line with previous findings showing that anti-CD25 aggravated acute allergic airway inflammation, ${ }^{22,27,60}$ but, it was ineffective in reversing the tolerant state. ${ }^{22}$ Using a OVA model of Th1and Th2-induced airway inflammation, Niu et al. ${ }^{26}$ showed that the vanishing of airway inflammation due to chronic OVA inhalation was associated with a population of TGF- $\beta$ expressing macrophages in the lungs. Indeed, we found that TGF- $\beta$ was expressed in the airways of acute and chronic mice, and that lung $\mathrm{CD} 11 \mathrm{c}^{+} \mathrm{MHCII}{ }^{+}$and $\mathrm{F} 4 / 80^{+} \mathrm{MHCII}^{+}$, but not $\mathrm{CD}^{+}{ }^{+} \mathrm{T}$ cells, expressed high levels of LAP. However, antiTGF- $\beta$ treatment failed to restore airway allergic inflammation of chronic mice, although it inhibited collagen formation. Our findings are in line with other studies showing that neutralization of TGF- $\beta$ abrogated airway remodeling. ${ }^{61,62}$

Apoptosis is another highly regulated pathway implicated in resolution of inflammatory processes and re-establishment of tissue homeostasis. We found that increased apoptosis of airway leukocytes, as revealed by annexin- $\mathrm{V}$ staining, was associated with the resolution of allergic inflammation. In chronic mice, we found that different cell types including eosinophils, neutrophils, $\mathrm{CD} 44^{+} \mathrm{T}$ cells, and antigen-presenting cells, such as $\mathrm{MHCII}^{+} \mathrm{CD} 11 \mathrm{~b}^{+}$cells, were undergoing apoptosis. In chronic group, the extrinsic pathway of apoptosis appeared to be operating, as the expression of apical casp8 and effector casp3 was only induced in this group. In contrast, the expression of casp9, an essential element in the intrinsic or mitochondrially gated pathway of apoptosis was augmented in acute group, reflecting probably a cytokine-induced mitochondrial stress. ${ }^{63}$ To further dissect the underlying apoptotic mechanism, we treated the mice with neutralizing $\mathrm{mAb}$ directed to key molecules responsible for death-inducing signals. The TRAIL pathway was found to be critically involved in apoptosis because anti-TRAIL, but not anti-FasL or anti-PD1 treatment restored allergic inflammation. Notably, antiTRAIL treatment augmented the frequency of activated lung Th2 cells. Keeping with this, previous study showed that frequency of lung $\mathrm{CD} 4{ }^{+} \mathrm{T}$ cells expressing the Th2 marker T1/ST2 decreased drastically after chronic OVA challenges. ${ }^{21}$ Conversely, treatment of allergic mice with rTRAIL decreased the frequency of Th2 cells producing IL-5. Although FasL expression was clearly augmented in chronic mice, anti-FasL treatment or Fas-deficiency did not affect the resolution of allergic inflammation. It was previously shown that Th2 cells preferentially express TRAIL, whereas Th1 cells express FasL. ${ }^{64}$ Our findings are in line with other studies showing the benefits of TRAIL signaling in tumors, ${ }^{65}$ bacterial meningitis, ${ }^{66}$ experimental autoimmune encephalitis, ${ }^{67}$ and neutrophilic inflammation. ${ }^{68}$

In humans, TRAIL binds to two death receptors (DR4/ TRAIL-R1 and DR5/TRAIL-R2), whereas in mice it binds to a single death receptor DR5, homolog to human TRAIL-R2. ${ }^{44}$ In asthmatic patients, survival of BAL eosinophils was prolonged after allergen provocation and this was correlated with increased expression of DcR2, a TRAIL decoy receptor, and decreased expressions of DR4 and DR5, the canonical human death receptors. ${ }^{69}$ Therefore, in humans the effect of TRAIL depends on the interactions and expression of five distinct receptors being detrimental when TRAIL binds to decoy receptors and beneficial when binding to death receptors. ${ }^{70}$ In a murine model of asthma, Weckmann et al. ${ }^{71}$ showed the role of TRAIL signaling in inducing pathognomic features of asthma, and more recently the same group extended their work by showing that TRAIL signaling in bronchial epithelium upregulated MID1 that promoted pro-allergic innate immune responses via inhibition of protein phosphatase $2 \mathrm{~A}^{40}$ In line with these findings, we also found high expression of TRAIL and MID1 in acute mice. Even though, we found that repeated IN administration of human rTRAIL, which preferentially activates mouse DR5, but not to mouse DcR1 or mouse DcR2 ${ }^{44}$ to allergic mice (acute group) increased apoptosis and attenuated Th2 responses and allergic lung inflammation, indicating that TRAIL treatment can overcome the relative resistance to apoptosis and induce the resolution of acute allergic inflammation. Our findings with rTRAIL conflict with previous study; ${ }^{40}$ however, the doses, administration, and animal model are different. We used four low doses of rTRAIL given to allergic mice, whereas previous work used a single-high dose given to naive mice. ${ }^{40}$ As mentioned before, TRAIL signaling does not always lead to apoptosis and in some 
circumstances non-apoptotic TRAIL signaling result in cell proliferation through the activation of nuclear factor-kappa B, protein kinase $\mathrm{B}$, c-Jun $\mathrm{N}$-terminal kinase, p38 mitogenactivated protein kinase, or induction of pro-allergic molecules. ${ }^{40,69,72}$ It remains to be determined how and when during the passage from acute to chronic OVA challenges proapoptotic molecules increase, whereas anti-apoptotic molecules decrease. Of note is the fact that two molecules involved with TRAIL signaling are differentially expressed in acute or chronic groups. MID1 expression (pro-allergic pathway) is increased in acute group, whereas insulin-like growth factorbinding protein-3, that potentiates TRAIL-induced apoptosis, is increased in chronic group. ${ }^{42}$ It follows that TRAIL signaling might have different outcomes according to the status of lung, naive non-challenged, acute or chronically challenged, and the balance of pro- and anti-apoptotic molecules. In fact, based on previous reports, the participation of death receptors and ligands that are members of the TNF receptor superfamily appears to have a dual role in lung inflammation because, depending of physiological or pathological conditions, they can promote inflammation or help in resolution of lung inflammation. ${ }^{68,71,73-75}$ Nevertheless, our data clearly demonstrate that TRAIL signaling is sufficient for downmodulation of allergic airway disease in mice, and suggest that deregulation of TRAIL-induced apoptosis could underline the chronic nature of asthma, emphasizing therefore a clinical promise of TRAIL and blockage of pro-allergic molecules in the treatment of asthma.

\section{METHODS}

Mice. Six- to-eight-week-old female BALB/c or IL-10KO mice were bred in the pathogen-free animal facility at the Institute of Biomedical Sciences of University of São Paulo. MRL/MpJ-Fas ${ }^{l p r}$ mice were provided by the School of Medicine of Ribeirão Preto and were housed at the Institute of Biomedical Sciences of University of São Paulo. The animal care committee of the University of São Paulo approved (number 002422) all procedures performed in this study.

Antigen sensitization and challenge. Mice were sensitized with $4 \mu \mathrm{g}$ of chicken OVA (grade V; Sigma, St Louis, MO) adsorbed to $1.6 \mathrm{mg}$ of alum $(\mathrm{Al}(\mathrm{OH}) 3)$ gel by subcutaneous route at days 0 and 7 . Chronic antigen exposure consisted of challenges with $10 \mu \mathrm{g}$ of OVA given IN once per week for 5 weeks at days 14,21, 28, 35, and 42. Acute exposure consisted of two IN OVA challenges starting at day 35 . In selected experiments, OVA challenges were given intraperitoneally. The experiments were performed $24 \mathrm{~h}$ after the last OVA challenge. For all experiments, LPS was removed from OVA by two to four cycles of Triton X-114 extractions. The endotoxin level of purified OVA after LPS depletion was below the limit of detection $\left(<0.01 \mathrm{ng} \mathrm{ml}^{-1}\right)$ as determined by Limulus amebocyte lysate QCL-1000 kit (BioWhittaker, Radnor, PA).

Cells. Mice were deeply anesthetized by IV injection of anesthetic solution containing ketamine and xylazine. For BAL cell collection, the tracheae were cannulated and lungs were lavaged with $1 \mathrm{ml}$ of PBS. For lung cell isolation, lungs were first perfused with PBS to remove residual blood. Lung tissue was then minced, digested with $2 \mathrm{mg} \mathrm{ml}^{-1}$ of collagenase type IV and $1 \mathrm{mg} \mathrm{ml}^{-1}$ of DNase (all from Sigma) for $30 \mathrm{~min}$ at $37^{\circ} \mathrm{C}$ and forced through a $70-\mu \mathrm{m}$ cell strainer to dissociate cells. The peritoneal cells were harvested by IP injection of $3 \mathrm{ml}$ of PBS. Total cell counts were determined by hemocytometer. Cytospin preparations of the BAL and peritoneal cells were stained with InstantProv (Newprov, Deerfield, IL), and differential cell counts were performed on 200 cells on the basis of morphology and staining.

Anti-CD25 treatment. Mice (acute and chronic) were treated with $500 \mu \mathrm{g}$ of purified anti-CD25 (PC61) mAb or with isotype control rat IgG1 (GL113) $\mathrm{mAb}$ or sterile PBS administered IV on days $34,35,36$, and 41 to deplete and/or to inactivate CD25 ${ }^{+}$putative Treg cells. ${ }^{27-30}$

Neutralizing mAb treatment. For blockage of death receptors signaling, chronic mice were treated with five IN administration of $50 \mu \mathrm{g}$ of neutralizing anti-TRAIL (N2B2), ${ }^{76}$ anti-FasL (MFL-4) ${ }^{77}$ or antiPD-1 (RMP1-14) ${ }^{78} \mathrm{mAb}$, twice at days 35 , once at day 36 , and twice at day 42. For anti-TGF- $\beta$ treatment, chronic mice received two IN injections of $30 \mu \mathrm{g}$ of anti-TGF- $\beta$ (1D11, R\&D Systems, Minneapolis, $\mathrm{MN}$ ) at days 34 and 41 .

rTRAIL treatment. Treatment with rTRAIL (SuperKillerTRAIL, Enzo Life Sciences, Farmingdale, NY) was performed in a well-established model of allergic airway disease induced by short-term allergen challenge as previously described. ${ }^{79}$ Briefly, mice were sensitized with $4 \mu \mathrm{g}$ of OVA in $1.6 \mathrm{mg}$ of alum at days 0 and 7 , and challenged with $10 \mu \mathrm{g}$ of OVA IN on days 14 and 21. During the period of antigen challenge, mice were treated with $1 \mu \mathrm{g}$ of rTRAIL or sterile PBS administered IN at days 14 and 15 and twice at day 21 .

Flow cytometry. Single-cell suspensions were stained with fluorochrome-conjugated antibodies against CD3 (145-2C11), CD4 (RM45), CD69 (H1.2F3), CD25 (PC61), Siglec-F (E50-2440), Gr1 (RB68C5), MHCII (M5/114.15.2), and LAP (TW7-16B4) (all from BD Biosciences, San Jose, CA) for $30 \mathrm{~min}$ at $4{ }^{\circ} \mathrm{C}$. For Foxp3 (FJK-16 s) and GATA-3 (L50-823) staining, cells were subsequently stained using the Foxp3 staining set (eBioscience, San Diego, CA) according to the manufacturer's protocol. For annexin- $\mathrm{V}$ and propidium iodide (PI) staining, cells were stained using the apoptosis kit (Calbiochem, San Diego, CA) according to the manufacturer's protocol. A Cytofix/ Cytoperm Plus kit with GolgiStop (BD Biosciences) were used for intracellular staining of IL-5 (TRFK5) and IL-4 (11B11) (BD Biosciences) according to the manufacturer's instructions. Data were acquired in a FACSCalibur or FACSCanto II (BD Biosciences) and analyzed with FlowJo software (TreeStar, Ashland, OR).

Cytokines and antibodies. Cytokines IL-4, IL-5, IL-10, interferon- $\gamma$ (BD Biosciences), IL-13 (R\&D Systems), and TGF- $\beta_{1}$ (Promega, Madison, WI) in the BAL fluid and total IgE (BD Biosciences) in the serum were measured using enzyme-linked immunosorbent assay kits according to the manufacturer's protocol. For determination of OVAspecific IgG1 and IgG2a, serum samples were plated on plates previously coated with OVA $\left(20 \mu \mathrm{g} \mathrm{ml}^{-1}\right.$; Sigma) and the bound antibodies were detected with goat anti-mouse IgG1 or IgG2a followed by peroxidase-labeled rabbit anti-goat IgG (all from Southern Biotechnology, Birmingham, AL). Purified mouse IgG1 (15H6) and IgG2a (HOPC-1, Southern Biotechnology) were used to estimate the concentration of OVA-specific antibody, as previously described. ${ }^{11}$

Lung histology. Lungs were prepared for histology by perfusing the animal by the right ventricle with $10 \mathrm{ml}$ of PBS. Lungs were then immersed in $10 \%$ phosphate-buffered formalin for $24 \mathrm{~h}$ followed by immersion in $70 \%$ ethanol until embedded in paraffin. Lung sections with $5 \mu \mathrm{m}$ were stained with periodic acid-Schiff (PAS)/hematoxylin for evaluation of inflammation and mucus production. The mucus index of PAS-stained bronchi areas was determined using the Metamorph 6.0 (Universal Images, Universal Imaging Corporation, Downingtown, PA) program.

Quantitative RT-PCR. Total RNA from lung cells was isolated with Trizol (Invitrogen, Carlsbad, CA). cDNA was synthesized by RevertAid $\mathrm{H}$ Minus M-MuLV-reverse transcriptase (Fermentas, Hanover, MD) and random primers. Real-time PCR analysis was 
performed on the StepOne Plus (Applied Biosystems, Foster City, CA) using SYBR green mix (Qiagen, Valencia, CA). Relative amounts of mRNA were normalized to $\beta$-actin RNA levels within each sample and expressed as relative target abundance by the $\Delta \Delta \mathrm{CT}$ method.

Statistical analysis. Statistical significance was assessed by either the two-tailed Student's $t$-test (two groups) or analysis of variance for multiple groups with a post hoc Tukey test to determine the significances ${ }^{\star} P<0.05,{ }^{\star *} P<0.01$, and ${ }^{\star * \star} P<0.001$, all performed using Prism 5.0 software (GraphPad, La Jolla, CA). Data are presented as mean \pm s.e.m.

SUPPLEMENTARY MATERIAL is linked to the online version of the paper at http://www.nature.com/mi

\section{ACKNOWLEDGMENTS}

This study was supported by Fundação de Amparo a Pesquisa do Estado de São Paulo (FAPESP) and Conselho Nacional de Desenvolvimento Científico e Tecnológico (CNPq). We thank Dr George dos Reis (Federal University of Rio de Janeiro) for advice, Silvia Bazin for assistance in RTPCR, and Paulo Albe for expert technical assistance in histological preparations.

\section{DISCLOSURE}

The authors declared no conflict of interest.

C 2014 Society for Mucosal Immunology

\section{REFERENCES}

1. Foster, P.S. et al. Elemental signals regulating eosinophil accumulation in the lung. Immunol. Rev. 179, 173-181 (2001).

2. Robinson, D.S. et al. Predominant TH2-like bronchoalveolar T-lymphocyte population in atopic asthma. N. Engl. J. Med. 326, 298-304 (1992).

3. Temann, U.A., Ray, P. \& Flavell, R.A. Pulmonary overexpression of IL-9 induces Th2 cytokine expression, leading to immune pathology. J. Clin. Invest. 109, 29-39 (2002).

4. Zhu, Z. et al. Pulmonary expression of interleukin-13 causes inflammation, mucus hypersecretion, subepithelial fibrosis, physiologic abnormalities, and eotaxin production. J. Clin. Invest. 103, 779-788 (1999).

5. Subbarao, P., Mandhane, P.J. \& Sears, M.R. Asthma: epidemiology, etiology and risk factors. CMAJ 181, E181-E190 (2009).

6. Durham, S.R. et al. Long-term clinical efficacy of grass-pollen immunotherapy. N. Engl. J. Med. 341, 468-475 (1999).

7. Jacobsen, L. et al. Specific immunotherapy has long-term preventive effect of seasonal and perennial asthma: 10-year follow-up on the PAT study. Allergy 62, 943-948 (2007).

8. Jacobsen, L., Wahn, U. \& Bilo, M.B. Allergen-specific immunotherapy provides immediate, long-term and preventive clinical effects in children and adults: the effects of immunotherapy can be categorised by level of benefit -the centenary of allergen specific subcutaneous immunotherapy. Clin. Transl. Allergy 2, 8 (2012).

9. Epstein, M.M. Do mouse models of allergic asthma mimic clinical disease? Int. Arch. Allergy Immunol. 133, 84-100 (2004).

10. Schroder, N.W. \& Maurer, M. The role of innate immunity in asthma: leads and lessons from mouse models. Allergy 62, 579-590 (2007).

11. Russo, M. et al. Suppression of asthma-like responses in different mouse strains by oral tolerance. Am. J. Resp. Cell Mol. Biol. 24, 518-526 (2001).

12. Keller, A.C. et al. Hierarchical suppression of asthma-like responses by mucosal tolerance. J. Allergy Clin. Immunol. 117, 283-290 (2006).

13. Mucida, D. et al. Oral tolerance in the absence of naturally occurring Tregs. J. Clin. Invest. 115, 1923-1933 (2005).

14. Mucida, D.S. et al. Unconventional strategies for the suppression of allergic asthma. Curr. Drug Targets Inflamm. Allergy 2, 187-195 (2003).

15. Yiamouyiannis, C.A. et al. Shifts in lung lymphocyte profiles correlate with the sequential development of acute allergic and chronic tolerant stages in a murine asthma model. Am. J. Pathol. 154, 1911-1921 (1999).
16. Swirski, F.K. et al. Chronic exposure to innocuous antigen in sensitized mice leads to suppressed airway eosinophilia that is reversed by granulocyte macrophage colony-stimulating factor. J. Immunol. 169, 3499-3506 (2002).

17. Sakai, K. et al. Prolonged antigen exposure ameliorates airway inflammation but not remodeling in a mouse model of bronchial asthma. Int. Arch. Allergy Immunol. 126, 126-134 (2001).

18. Van Hove, C.L. et al. Prolonged inhaled allergen exposure can induce persistent tolerance. Am. J. Respir. Cell Mol. Biol. 36, 573-584 (2007).

19. Schramm, C.M. et al. Chronic inhaled ovalbumin exposure induces antigen-dependent but not antigen-specific inhalational tolerance in a murine model of allergic airway disease. Am. J. Pathol. 164, 295-304 (2004).

20. Kabbur, P.M. et al. Interleukin-10 does not mediate inhalational tolerance in a chronic model of ovalbumin-induced allergic airway disease. Cell Immunol. 239, 67-74 (2006).

21. Swirski, F.K. et al. Prolonged ovalbumin exposure attenuates airway hyperresponsiveness and Tcell function in mice. Int. Arch. Allergy Immunol. 141, 130-140 (2006).

22. Carson, W.F. et al. Accumulation of regulatory T cells in local draining lymph nodes of the lung correlates with spontaneous resolution of chronic asthma in a murine model. Int. Arch. Allergy Immunol. 145, 231-243 (2008).

23. Strickland, D.H. et al. Reversal of airway hyperresponsiveness by induction of airway mucosal CD4 + CD25 + regulatory T cells. J. Exp. Med. 203, 2649-2660 (2006).

24. Burchell, J.T. et al. Attenuation of allergen-induced airway hyperresponsiveness is mediated by airway regulatory Tcells. Am. J. Physiol. Lung Cell Mol. Physiol. 296, L307-L319 (2009).

25. Singh, A. et al. Regulatory role of B cells in a murine model of allergic airway disease. J. Immunol. 180, 7318-7326 (2008).

26. Niu, N. et al. A novel pathway that regulates inflammatory disease in the respiratory tract. J. Immunol. 178, 3846-3855 (2007).

27. Faustino, L. et al. Regulatory T cells migrate to airways via CCR4 and attenuate the severity of airway allergic inflammation. J. Immunol. 190, 2614-2621 (2013).

28. Kohm, A.P. et al. Cutting Edge: Anti-CD25 monoclonal antibody injection results in the functional inactivation, not depletion, of $\mathrm{CD} 4+\mathrm{CD} 25+$ T regulatory cells. J. Immunol. 176, 3301-3305 (2006).

29. Couper, K.N. et al. Incomplete depletion and rapid regeneration of Foxp3 + regulatory $\mathrm{T}$ cells following anti-CD25 treatment in malariainfected mice. J. Immunol. 178, 4136-4146 (2007).

30. Setiady, Y.Y., Coccia, J.A. \& Park, P.U. In vivo depletion of CD4 + FOXP3 + Treg cells by the PC61 anti-CD25 monoclonal antibody is mediated by FcgammaRIII + phagocytes. Eur. J. Immunol. 40, 780-786 (2010).

31. Bedoret, D. et al. Lung interstitial macrophages alter dendritic cell functions to prevent airway allergy in mice. J. Clin. Invest. 119, 3723-3738 (2009).

32. Joetham, A. et al. Naturally occurring lung CD4(+)CD25(+) T cell regulation of airway allergic responses depends on IL-10 induction of TGFbeta. J. Immunol. 178, 1433-1442 (2007).

33. Taylor, A.W. Review of the activation of TGF-beta in immunity. J. Leukoc. Biol. 85, 29-33 (2009).

34. Halwani, R. et al. Role of transforming growth factor-beta in airway remodeling in asthma. Am. J. Respir. Cell Mol. Biol. 44, 127-133 (2011).

35. Tong, J. et al. Fas-positive $T$ cells regulate the resolution of airway inflammation in a murine model of asthma. J. Exp. Med. 203, 1173-1184 (2006).

36. McGrath, E.E. et al. Deficiency of tumour necrosis factor-related apoptosis-inducing ligand exacerbates lung injury and fibrosis. Thorax 67, 796-803 (2012).

37. Aggarwal, B.B. Signalling pathways of the TNF superfamily: a doubleedged sword. Nat. Rev. Immunol. 3, 745-756 (2003).

38. Rossi, D. \& Gaidano, G. Messengers of cell death: apoptotic signaling in health and disease. Haematologica 88, 212-218 (2003).

39. Ishida, $Y$. et al. Induced expression of PD-1, a novel member of the immunoglobulin gene superfamily, upon programmed cell death. EMBO J. 11, 3887-3895 (1992).

40. Collison, A. et al. The E3 ubiquitin ligase midline 1 promotes allergen and rhinovirus-induced asthma by inhibiting protein phosphatase 2A activity. Nat. Med. 19, 232-237 (2013). 
41. Grimberg, A. et al. IGFBP-3 mediates p53-induced apoptosis during serum starvation. Int. J. Oncol. 21, 327-335 (2002).

42. Williams, A.C. et al. Insulin-like growth factor binding protein 3 (IGFBP-3) potentiates TRAlL-induced apoptosis of human colorectal carcinoma cells through inhibition of NF-kappaB. Cell Death Differ. 14, 137-145 (2007).

43. Johnson, C.R. \& Jarvis, W.D. Caspase-9 regulation: an update. Apoptosis 9, 423-427 (2004).

44. Schneider, P. et al. Identification of a new murine tumor necrosis factor receptor locus that contains two novel murine receptors for tumor necrosis factor-related apoptosis-inducing ligand (TRAIL). J. Biol. Chem. 278 5444-5454 (2003)

45. Davidson, N.J. et al. Chronic colitis in IL-10 - / - mice: insufficient counter regulation of a Th1 response. Int. Rev. Immunol. 19, 91-121 (2000).

46. Christ, M. et al. Immune dysregulation in TGF-beta 1-deficient mice. J. Immunol. 153, 1936-1946 (1994).

47. Kantarci, A. \& Van Dyke, T.E. Lipoxins in chronic inflammation. Crit. Rev. Oral Biol. Med. 14, 4-12 (2003).

48. Moran, M. \& Nicholson, P. Prostaglandin deficiency. Prostaglandins Leukot. Essent. Fatty Acids 39, 83-89 (1990).

49. Barnes, P.J. Anti-inflammatory actions of glucocorticoids: molecular mechanisms. Clin. Sci. 94, 557-572 (1998).

50. Wang, $\mathrm{H}$. et al. Nicotinic acetylcholine receptor alpha7 subunit is an essential regulator of inflammation. Nature 421, 384-388 (2003).

51. Shevach, E.M. From vanilla to 28 flavors: multiple varieties of T regulatory cells. Immunity 25, 195-201 (2006).

52. Mauri, C. \& Bosma, A. Immune regulatory function of B cells. Annu. Rev. Immunol. 30, 221-241 (2012).

53. Fleming, B.D. \& Mosser, D.M. Regulatory macrophages: setting the threshold for therapy. Eur. J. Immunol. 41, 2498-2502 (2011).

54. Chen, X., Armstrong, M.A. \& Li, G. Mesenchymal stem cells in immunoregulation. Immunol. Cell Biol. 84, 413-421 (2006).

55. Savill, J. Apoptosis in resolution of inflammation. J. Leukoc. Biol. 61, 375-380 (1997).

56. Haslett, C. et al. Granulocyte apoptosis and the control of inflammation. Philos. Trans. R Soc. Lond. B Biol. Sci. 345, 327-333 (1994).

57. Kankaanranta, H. et al. Delayed eosinophil apoptosis in asthma. J. Allergy Clin. Immunol. 106, 77-83 (2000).

58. Maret, M. et al. A role for Bid in eosinophil apoptosis and in allergic airway reaction. J. Immunol. 182, 5740-5747 (2009).

59. de Souza, P.M. \& Lindsay, M.A. Apoptosis as a therapeutic target for the treatment of lung disease. Curr. Opin. Pharmacol. 5, 232-237 (2005).

60. Saito, K. et al. Differential regulatory function of resting and preactivated allergen-specific CD4 + CD25 + regulatory T cells in Th2-type airway inflammation. J. Immunol. 181, 6889-6897 (2008).

61. Alcorn, J.F. et al. Transforming growth factor-beta1 suppresses airway hyperresponsiveness in allergic airway disease. Am. J. Respir. Crit. Care Med. 176, 974-982 (2007).
62. McMillan, S.J., Xanthou, G. \& Lloyd, C.M. Manipulation of allergen-induced airway remodeling by treatment with anti-TGF-beta antibody: effect on the Smad signaling pathway. J. Immunol. 174, 5774-5780 (2005).

63. Thomas, H.E. \& Biden, T.J. Bad news for beta-cell apoptosis. Diabetes $\mathbf{5 8}$, 1725-1727 (2009).

64. Zhang, X.R. et al. Reciprocal expression of TRAIL and CD95L in Th1 and Th2 cells: role of apoptosis in T helper subset differentiation. Cell Death Differ. 10, 203-210 (2003).

65. Grosse-Wilde, A. et al. TRAIL-R deficiency in mice enhances lymph node metastasis without affecting primary tumor development. J. Clin. Invest. 118, 100-110 (2008).

66. Hoffmann, O. et al. TRAIL limits excessive host immune responses in bacterial meningitis. J. Clin. Invest. 117, 2004-2013 (2007).

67. Cretney, E. et al. TNF-related apoptosis-inducing ligand (TRAIL)/Apo2L suppresses experimental autoimmune encephalomyelitis in mice. Immunol. Cell Biol. 83, 511-519 (2005).

68. McGrath, E.E. et al. TNF-related apoptosis-inducing ligand (TRAIL) regulates inflammatory neutrophil apoptosis and enhances resolution of inflammation. J. Leukoc. Biol. 90, 855-865 (2011).

69. Robertson, N.M. et al. Differential expression of TRAIL and TRAIL receptors in allergic asthmatics following segmental antigen challenge: evidence for a role of TRAIL in eosinophil survival. J. Immunol. 169, 5986-5996 (2002).

70. MacFarlane, M. TRAIL-induced signalling and apoptosis. Toxicol. Lett. 139, 89-97 (2003).

71. Weckmann, M. et al. Critical link between TRAIL and CCL20 for the activation of $\mathrm{TH} 2$ cells and the expression of allergic airway disease. Nat. Med. 13, 1308-1315 (2007).

72. Lorz, C. et al. Trail and kidney disease. Front Biosci. 14, 3740-3749 (2009).

73. Borges, V.M. et al. Fas ligand triggers pulmonary silicosis. J. Exp. Med. 194, 155-164 (2001).

74. Hameed, A.G. et al. Inhibition of tumor necrosis factor-related apoptosisinducing ligand (TRAIL) reverses experimental pulmonary hypertension. J. Exp. Med. 209, 1919-1935 (2012).

75. Steinwede, K. et al. TNF-related apoptosis-inducing ligand (TRAIL) exerts therapeutic efficacy for the treatment of pneumococcal pneumonia in mice. J. Exp. Med. 209, 1937-1952 (2012).

76. Kayagaki, N. et al. Involvement of TNF-related apoptosis-inducing ligand in human CD4 + T cell-mediated cytotoxicity. J. Immunol. 162, 2639-2647 (1999).

77. Kayagaki, N. et al. Polymorphism of murine Fas ligand that affects the biological activity. Proc. Natl. Acad. Sci. USA 94, 3914-3919 (1997).

78. Yamazaki, T. et al. Blockade of B7-H1 on macrophages suppresses CD4 + Tcell proliferation by augmenting IFN-gamma-induced nitric oxide production. J. Immunol. 175, 1586-1592 (2005).

79. Faustino, L. et al. Regulatory T cells accumulate in the lung allergic inflammation and efficiently suppress T-cell proliferation but not Th2 cytokine production. Clin. Dev. Immunol. 2012, 721817 (2012). 